\title{
Relationship Between Intrinsic Threshold Stress Intensity And Near-Tip Residual Stress: A Reference Material Property
}

\author{
R Sunder ${ }^{1}$
}

${ }^{1}$ Bangalore Integrated System Solutions Pvt Ltd

July 5,2021

\begin{abstract}
The relationship between intrinsic, closure-free threshold stress intensity and near-tip residual stress, characterizes the effect of load magnitude as well as load history on near-threshold fatigue crack growth rates. It serves as a reference against which precise closure data can be extracted from growth rates to calibrate analytical estimates. These possibilities were subjected to rigorous experimental verification involving threshold and near-threshold fatigue response under overloads, underloads with load-shedding on a steel prone to oxide debris formation. The study reveals why conventional load shedding practice to characterize threshold stress intensity is prone to yield unconservative and misleading results.
\end{abstract}

\section{Hosted file}

IntrinsicThresholdStressIntensityAsReference.docx available at https://authorea.com/users/ 302203/articles/529015-relationship-between-intrinsic-threshold-stress-intensity-andnear-tip-residual-stress-a-reference-material-property 